\title{
MIXING IN STARS
}

(Abstract)

PETER P. EGGLETON

Institute of Astronomy, Cambridge, U.K.

Mixing of composition in a star may be due not only to convection and semiconvection but also to circulation, as Prof. Kippenhahn has reminded us, and perhaps also to instability, for instance as Dilke and Gough (1972) have suggested for the Sun. I have tried to show in the past that convective and semiconvective mixing can be described by a diffusion equation

$$
\frac{\partial}{\partial m} \sigma \frac{\partial X}{\partial m}=\frac{D X}{D t}+X R
$$

where $\sigma$ is a suitable mixing rate, e.g.

$$
\sigma \propto\left(\mathrm{V}_{r}-\mathrm{V}_{a}\right)^{2}
$$

or

$$
\sigma \propto\left(\nabla_{r}-\nabla_{a}-\frac{\beta}{4-3 \beta} \frac{\mathrm{d} \log \mu}{\mathrm{d} \log p}\right)^{2}
$$

in an unstable region. Of course $\sigma=0$ in a stable region. Provided equation (1) is solved simultaneously with the structure equations condition (2A) readily leads to convection and to Schwarzschild's semiconvection. I have not used (2B), but I suspect that in at least some cases it may not lead to a unique answer.

Recently Dr J. Perdang and I have tried using Equation (1) to describe a hypothetical mixing that might arise in and near a shell source in a red giant. We suppose there may be an instability, perhaps of a non-radial gravitational mode which grows on a thermal time scale, whose overall effect on the evolution of the star may be described qualitatively as a diffusion of composition on a thermal timescale. This suggests that we take a mixing rate

$$
\sigma \sim\left(4 \pi r^{2} \varrho\right)^{2}\left(l^{2} / \tau\right)
$$

with $l$ being a typical length scale for the unstable mode and $\tau$ being a thermal time scale. With such a mixing we find that the luminosity of a red giant increases rapidly.

A. J. C. Bolton and I have used an equivalent analysis to re-examine the stability of thin burning shells to spherical perturbations on a thermal time scale. Although our equilibrium model of such shells is rather crude, and not applicable to all kinds of burning shells that may actually occur, the perturbation analysis of the model was carried out exactly. Our conclusion is broadly that the stability of a shell, as well as 
its structure, is mainly determined by $\eta$, where

$$
\eta \equiv\left(\frac{\partial \log \varepsilon_{\mathrm{nuc}}}{\partial \log T}\right)_{X, p} .
$$

For hydrogen shells instability (with a complex eigenvalue) sets in when $\eta \geqslant 14$; for helium burning, when $\eta \geqslant 24$. This suggests that even hydrogen burning shells, particularly in their early stages, may be unstable by a narrow margin.

Gough and Dilke (1972) have shown that the Sun, which is generally found to be stable to radial thermal instabilities, may be unstable to certain dynamical modes ( $g$-modes) which grow on a thermal timescale. Their work may be applicable to all stars where burning takes place in a radiative region, e.g. stars with thin shells, and I think there is a strong possibility that all such shells are unstable on a thermal time scale to such non-radial dynamical modes.

In the late stages of stellar evolution the thermal and nuclear timescales in the central regions may be not very different, and the perturbation of the composition profile may not be negligible. Equation (1), when solved along with the conventional structure equations, should allow this effect to be easily included.

\section{Reference}

Dilke, F. W. W. and Gough, D. O.: 1972, Nature 240, 262. 